\title{
Femtosecond photoelectron spectroscopy of the photodissociation of $\mathrm{Au}_{3}^{-}$
}

\author{
G. Ganteför*, S. Kraus, W. Eberhardt \\ Institut für Festkörperforschung, Forschungszentrum Jülich,D-52425 Jülich, Germany
}

\begin{abstract}
The photodissociation of $\mathrm{Au}_{3}^{-}$is studied by time-resolved photoelectron spectroscopy using femtosecond laser pulses. The spectra show the time evolution of the electronic structure after photoabsorption. Absorption of a "pump" photon results in the formation of a metastable activated complex $\left(\mathrm{Au}_{3}^{-}\right)^{*}$, which decays by dissociation into $\mathrm{Au}_{1}^{-}+\mathrm{Au}_{2}$ and, less favorably, into $A u_{2}^{-}+\mathrm{Au}_{1}$. Photoelectron spectra recorded with the "probe" laser pulse at increasing delay time first show the spectrum of the intact $\mathrm{Au}_{3}^{-}$, then that of the activated complex $\left(\mathrm{Au}_{3}^{-}\right)^{*}$ and, finally, the spectra of the two charged dissociation products $\mathrm{Au}_{1}^{-}$ and $\mathrm{Au}_{2}^{-}$. The lifetime of $\left(\mathrm{Au}_{3}^{-}\right)^{*}$ decreases dramatically with increasing energy of the "pump" photon. (C) 1998 Elsevier Science B.V.
\end{abstract}

Keywords: Photoelectron spectroscopy; Photodissociation; $\mathrm{Au}_{3}^{-}$

Femtosecond lasers with pulse lengths of typically $100 \mathrm{fs}$ or less open the way to the study of fast dynamic processes [1-3]. A basic process of this type is the change in the electronic structure of a molecule in a chemical reaction or upon dissociation. Information about the dynamics of the molecular orbitals can be gained by some elegant indirect methods $[4,5]$, but a direct picture of the time evolution is obtained by the use of time-resolved techniques only. One textbook example is the study of the wavepacket motion of nucleii in bound electronic states $[3,6,7]$ of a molecule. Spectroscopy of molecular orbitals during dissociation has been conducted using different techniques such as laser-induced fluorescence and mass spectroscopy $[8,9]$. However, photoelectron spectroscopy gives a direct insight into the electronic structure and, therefore, time-resolved photoelectron spectroscopy is an ideal tool to study

* Corresponding author. the time evolution of the electronic structure during an "inverse" chemical reaction (dissociation).

Here, we apply femtosecond time-resolved photoelectron spectroscopy (TRPES) to the study of the photodissociation of $\mathrm{Au}_{3}^{-}$. We show that in this specific molecule the dissociation mechanism depends on the excitation energy and is either a fast direct process or a process that occurs via a long-lived activated complex. The activated complex gives rise to the appearance of new features in the photoelectron spectra that are different from those of the parent and fragment molecules. In addition, by the choice of the photon energy we are able to alter the lifetime of the complex and its dissociation pathways.

TRPES experiments have been carried out to study electronic relaxation processes in stable molecules [10-12] and solids [13,14]. Femtosecond lasers have also been combined with zero electron kinetic energy spectroscopy [15,16]. Recently, TRPES has been applied to the study of negatively charged species. 
Greenblatt et al. [17] have studied the photodissociation of $\mathrm{I}_{2}^{-}$, which dissociates as a direct process within 100 fs. During dissociation the photoemission peaks of the fragment $I_{1}^{-}$show an asymetric broadening, which is related to the shapes of the potential curves of the electronic states involved. Recently the same dissociation process has been studied for an $\mathrm{I}_{2}^{-}$molecule embedded in a solvation shell [18].

The application of TRPES to negatively charged species has two advantages.

1. The electron can be removed in a one-photon process even at the relatively low photon energies available from typical femtosecond lasers (below $6 \mathrm{eV}$ ). Furthermore, with a photon energy of $3.49 \mathrm{eV}$ already the whole valence band structure of small $\mathrm{Na}_{n}^{-}$clusters can be revealed [19]. Spectra recorded for one-photon events can be easily analyzed in contrast to spectra obtained by multiphoton ionization.

2. For the study of clusters, mass separation prior to the experiment is essential. Therefore, photoelectron spectroscopy of negatively charged species using standard lasers has been successfully applied to the study of the valence band stucture of clusters (see Ref. [20] and references cited therein). In addition, photoelectron spectra contain information about the final states, which are the neutral ground and excited states in the case of an anion. Calculations usually refer to neutral states.

The experimental set-up using standard nanosecond pulsed lasers has been described in detail elsewhere [20]. The negatively charged clusters are produced with a pulsed arc cluster ion source and cooled down to approximately room temperature in a seed gas $(\mathrm{He})$. The anions are accelerated in a pulsed electric field and, depending on their time of flight, the clusters separate into a chain of bunches of defined cluster size. The kinetic energy of electrons detached from a selected bunch by a UV-laser pulse is measured using a "magnetic bottle" time-of-flight electron spectrometer. For the time-resolved experiment, the single UV laser is replaced by two femtosecond pulses, the pump and the probe pulse, separated by a tunable delay $(0-3600 \mathrm{ps})$. The photon energy of both pulses is equal and tunable between 3.0 and $3.14 \mathrm{eV}$. The pulse energy of the pump pulse is about five times higher than that of the probe pulse. This results in the

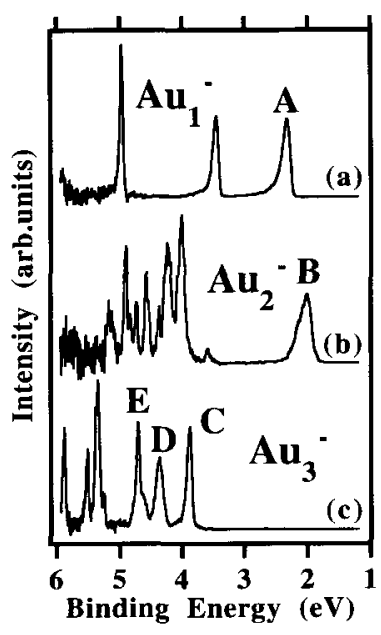

Fig. 1. Photoelectron spectra of (a) $\mathrm{Au}_{1}^{-}$, (b) $\mathrm{Au}_{2}^{-}$and (c) $\mathrm{Au}_{3}^{-}$ obtained using a standard UV laser with a photon energy of $h v=6.4 \mathrm{eV}$. For a discussion of the marked features, see text.

maximum pump/probe signal. The photon flux of the pump pulse is kept as low as possible (below $0.1 \mathrm{~mJ} \mathrm{~cm}^{-2}$ ) to minimize the two-photon detachment signal (see below). The time resolution is better than $230 \mathrm{fs}$.

There are two photodissociation pathways of the $\mathrm{Au}_{3}^{-}[21]$ :

$\mathrm{Au}_{3}^{-}+h \nu \rightarrow \mathrm{Au}_{1}^{-}+\mathrm{Au}_{2}$

$\mathrm{Au}_{3}^{-}+h \nu \rightarrow \mathrm{Au}_{2}^{-}+\mathrm{Au}_{1}$

The additional electron may reside on the atom or dimer fragment. For comparison, Fig. 1 displays photoelectron spectra of all species $\left(\mathrm{Au}_{1}^{-}, \mathrm{Au}_{2}^{-}, \mathrm{Au}_{3}^{-}\right)$ that possibly contribute to the pump/probe photoemission signal. These have been obtained using a standard ArF excimer laser at a photon energy of $h \nu=6.4 \mathrm{eV}$. The spectra give an overview of the electronic structure of $\mathrm{Au}_{3}^{-}$(ionization potential IP, $3.9 \mathrm{eV}), \mathrm{Au}_{2}^{-}(\mathrm{IP}, 2.0 \mathrm{eV})$ and $\mathrm{Au}_{1}^{-}(\mathrm{IP}, 2.3 \mathrm{eV})$. From the neutral fragments $\left(\mathrm{Au}_{1}, \mathrm{Au}_{2}\right)$ there are no photoelectron signals, because the IPs are too high.

During the dissociation process, all spectral features undergo changes. We focus here on the lowest binding energy structures (Fig. 1, marked $\mathrm{A}$ for $\mathrm{Au}_{1}^{-}$, $\mathrm{B}$ for $\mathrm{Au}_{2}^{-}$and $\mathrm{C}$ for $\mathrm{Au}_{3}^{-}$), because of the low photon energy of the probe pulse in our time-resolved experiment $(h \nu=3.0 \mathrm{eV})$. From an analysis of the electronic structure [22] we expect that feature $\mathrm{C}$ of $\mathrm{Au}_{3}^{-}$ 


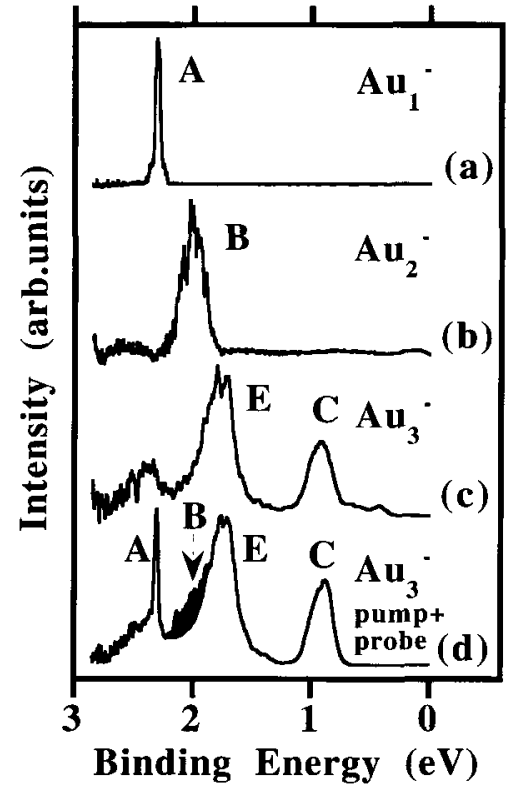

Fig. 2. Photoelectron spectra of (a) $\mathrm{Au}_{1}^{-}$, (b) $\mathrm{Au}_{2}^{-}$and (c) $\mathrm{Au}_{3}^{-}$ obtained using the pump pulse $(h \nu=3.0 \mathrm{eV})$ only. The lowest spectrum (d) is a pump/probe photoelectron spectrum with a delay of $3.6 \mathrm{~ns}$ between the two laser pulses $\left(h \nu_{\text {pump }}=h \nu_{\text {probe }}=3.0 \mathrm{eV}\right)$. For a discussion of the marked features, see text.

develops into peaks $\mathrm{A}$ of $\mathrm{Au}_{1}^{-}$(process shown as Eq. (1)) or $\mathrm{B}$ of $\mathrm{Au}_{2}^{-}$(process shown as Eq. (2)), respectively. During dissociation, the peaks may shift and possibly split or merge while forming the spectrum of the fragment. (This is, however, a crude simplification neglecting, for example, electronic relaxation, Frank-Condon profiles, multiplet splitting and shake up processes.) In addition, at intermediate times, while the fragments are still interacting electronically, new features might appear in the spectra.

In Fig. 2, examples of photoelectron spectra of $\mathrm{Au}_{1}^{-}$, $\mathrm{Au}_{2}^{-}$and $\mathrm{Au}_{3}^{-}$obtained with femtosecond laser pulses are displayed. The upper three spectra (Fig. 2(a)-(c)) of $\mathrm{Au}_{1}^{-}, \mathrm{Au}_{2}^{-}$and $\mathrm{Au}_{3}^{-}$are recorded using the pump pulse $(h \nu=3.0 \mathrm{eV})$ only. The spectra of $\mathrm{Au}_{1}^{-}$and $\mathrm{Au}_{2}$ correspond to those shown in Fig. 1, taking the higher photon energy $(h \nu=6.4 \mathrm{eV})$ into consideration. According to Fig. 1, no photoemission signal is to be expected for $\mathrm{Au}_{3}^{-}$at photon energies lower than $3.9 \mathrm{eV}$. However, two features (Fig. 2(c)) at binding energies of $0.9 \mathrm{eV}$ (marked C) and $1.7 \mathrm{eV}$ (marked E) are observed. These are assigned to a two-photon detachment process $(h \nu=2 \times 3 \mathrm{eV})$ and correspond to the features marked $\mathrm{C}$ and $\mathrm{E}$ in Fig. 1 at the binding energies $3.9 \mathrm{eV}(0.9+3.0 \mathrm{eV})$ and $4.7 \mathrm{eV}(1.7+$ $3.0 \mathrm{eV}$ ), respectively. Feature D (Fig. 1(c)) is not observed in Fig. 2(c), which might be caused by the different selection rules governing two-photon photodetachment. No such two-photon detachment processes are observed for $\mathrm{Au}_{1}^{-}$and $\mathrm{Au}_{2}^{-}$. The reason is the large cross-section for photodetachment compared to photoexcitation. For $\mathrm{Au}_{1}^{-}$and $\mathrm{Au}_{2}^{-}$, singlephoton detachment is allowed and photoexcitation is suppressed. For $\mathrm{Au}_{3}^{-}$, single-photon detachment is not possible for energy reasons. Detachment from a photoexcited species, on the other hand, can be observed. Therefore, photoexcitation will immediately result in detachment by a second photon.

The lower trace in Fig. 2(d) shows a pump/probe photoelectron spectrum of $\mathrm{Au}_{3}^{-}$at the maximum delay attainable in our experiment (3.6 ns). An additional narrow peak at $2.3 \mathrm{eV}$ binding energy (marked A) is observed. The feature is assigned to $\mathrm{Au}_{1}^{-}$produced by the photodissociation of $\mathrm{Au}_{3}^{-}$. In addition, a shoulder at the low binding energy side of feature $E$ (marked $B$ ) appears, which is assigned to $\mathrm{Au}_{2}^{-}$fragments.

We studied the time dependence of the dissociation process Eq. (1) corresponding to the appearance of peak A (Fig. 2(d)) in detail. Fig. 3 shows an expanded view of the high binding energy range of a series of TRPES spectra of $\mathrm{Au}_{3}^{-}$recorded at varying time delays between the pump and probe pulse. The spectra are normalized to the two-photon detachment signal of the (more intense) pump pulse (Fig. 2(c)), which is always superimposed to the pump/probe signal. The spectra labeled 0.0 (equal to a 0.0 ps delay) and 3600 (equal to a 3600 ps delay) correspond to the "stationary" spectra of $\mathrm{Au}_{3}^{-}$and $\mathrm{Au}_{1}^{-}$displayed in Fig. 2(c) and (a), respectively. At time zero there is no significant signal in the energy window shown in Fig. 3, indicating that $\mathrm{Au}_{3}^{-}$is still intact. Already after $0.6 \mathrm{ps}$, a broad maximum (hatched) appears, which develops with increasing time into the narrow peak characteristic of $\mathrm{Au}_{1}^{-}$(spectrum at top, see also feature A in Fig. 1(a) and Fig. 2(a)). For intermediate times $(200-3600 \mathrm{ps})$, the feature consists of two components: a broad maximum (hatched) and a superimposed narrow peak (black). The narrow peak increases gradually in intensity, while the broad feature weakens. 


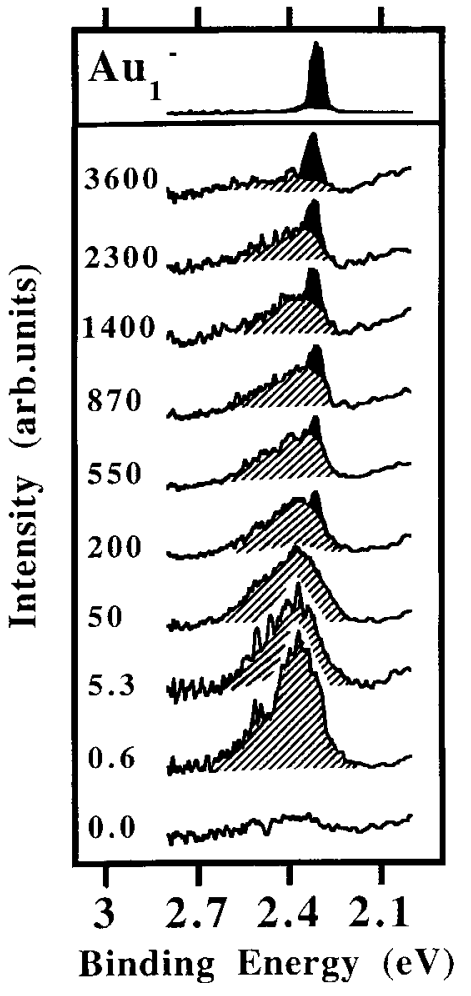

Fig. 3. Series of pump/probe photoelectron spectra recorded with a photon energy of $h \nu=3.0 \mathrm{eV}$. Only the binding energy range of the $\mathrm{Au}^{-}$fragment peak $\mathrm{A}$ is displayed. The time resolution is $230 \mathrm{fs}$. The numbers are the pump/probe delays given in picoseconds. The uppermost trace is a spectrum of $\mathrm{Au}_{1}^{-}$similar to the one shown in Fig. 1a, but recorded with a photon energy of $h \nu=3.0 \mathrm{eV}$. The broad feature (hatched) is assigned to photoemission from the activated complex $\mathrm{Au}_{3}^{-*}$ and the superimposed narrow peak (black) corresponds to emission from the $\mathrm{Au}_{1}^{-}$fragment.

The series of spectra displayed in Fig. 3 can be interpreted assuming the existence of a metastable activated complex corresponding to an excited state of $\mathrm{Au}_{3}^{-}$. Thus we have to modify the two dissociation channels discussed above by including the metastable state

$\mathrm{Au}_{3}^{-}+h \nu \rightarrow\left(\mathrm{Au}_{3}^{-*}\right) \rightarrow \mathrm{Au}_{1}^{-}+\mathrm{Au}_{2}$

$\mathrm{Au}_{3}^{-}+h \nu \rightarrow\left(\mathrm{Au}_{3}^{-*}\right) \rightarrow \mathrm{Au}_{2}^{-}+\mathrm{Au}_{1}$

Photodetachment from this excited state of $\mathrm{Au}_{3}^{-}$gives rise to the broad feature (hatched) visible in Fig. 3.

Fig. 4 displays a schematic of speculative potential curves of the two electronic states involved in the photodissociation process $(\mathrm{X}$, ground state; $\mathrm{A}$, excited

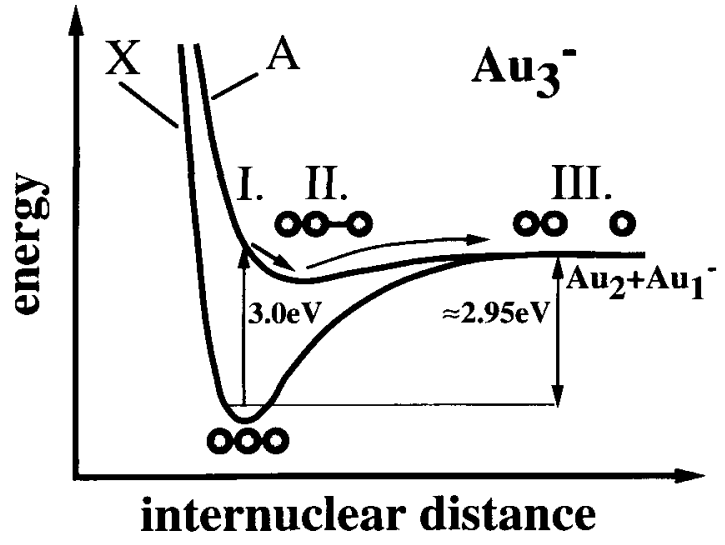

Fig. 4. Scheme of speculative potential curves of the two electronic states involved in the photodissociation process $\mathrm{Eq}$. (3) (X, ground state; $\mathrm{A}$, excited state). The ground state of $\mathrm{Au}_{3}^{-}$is probably linear. After photoexcitation the molecule passes through three stages (I, II and III), as indicated by the arrows. These correspond to the three different types of photoelectron spectra of $\mathrm{Au}_{3}^{-}$(I, Fig. 2c; II, Fig. 3 at 0.6 ps delay; III, Fig. 2 d).

state). After photoexcitation, the molecule passes through three stages (I, II, III), which correspond to the three different photoelectron spectra recorded.

Stage I (delay $0.0 \mathrm{ps}$ ). The excitation process is fast compared to the movement of the nucleii. Therefore at time zero, the excited molecule $\mathrm{Au}_{3}^{-*}$ still has the geometric structure of ground state $\mathrm{Au}_{3}^{-}$. Photodetachment from this species ends up in the same final states (Fig. 2(c), features C, E) as the one-photon detachment process at $6.4 \mathrm{eV}$ photon energy shown in Fig. 1(a) (features C, E). Therefore, the photoelectron spectra are similar apart from differences in the peak intensities due to the different transition probabilities involving the intermediate state. This is the two-photon detachment process mentioned above, which is, according to Fig. 4, a resonant process.

Stage II (delay less than $0.2 \mathrm{ps}$ ). The molecule relaxes into the new equilibrium geometry of the excited state, which presumably has a different electronic structure. For example, the electron affinity increases from $0.9 \mathrm{eV}$ of the unrelaxed excited state to roughly $2.4 \mathrm{eV}$. This process occurs on a time-scale comparable to our experimental resolution (230 fs) and corresponds to the appearance of the new transient emission feature (Fig. 3, hatched). The geometry of the activated complex might correspond to an 
"almost" dissociated trimer with one extended bond, as indicated in Fig. 4.

Stage III (delay less than $0.1 \mathrm{~ns}$ ). The excess energy stored in the vibrational degrees of freedom of $\mathrm{Au}_{3}^{-*}$ is sufficient to break the extended bond. The process is thermally activated in a similar way to the unimolecular dissociation of larger clusters. Therefore, the dissociation of one individual molecule might happen at any time greater than $0.2 \mathrm{~ns}$. The statistical nature of this process results in the slow "growth" of the $\mathrm{Au}_{1}^{-}$fragment peak (Fig. 3, black) and the corresponding decrease of the transient feature (Fig. 3, hatched). The lifetime of $\mathrm{Au}_{3}^{-*}$ can be determined from Fig. 3 and is $1500 \pm 200$ ps.

The lifetime must be strongly (exponentially) dependent on the excess energy. Indeed, at only a $140 \mathrm{meV}$ higher photon energy of the pump pulse the dissociation process is altered dramatically as follows.

- In a preliminary experiment we determined an upper limit of the lifetime of the activated complex of less than $50 \mathrm{ps}$.

- The branching ratio between the two possible decay channels (eqns (3) and (4)) is altered by a factor of 2.5 .

From the first observation we estimate the threshold for dissociation to be $2.95 \mathrm{eV}$ (Fig. 4). To demonstrate the change in the branching ratios between the two dissociation channels, Fig. 5(a) displays the difference spectrum obtained by subtracting the pump/probe photoelectron spectrum of $\mathrm{Au}_{3}^{-}$recorded at zero delay $(\Delta t=0.0 \mathrm{ps})$ from that obtained at maximum delay ( $\Delta t=3600 \mathrm{ps}$ ). By this procedure, the emission signals from the fragments $\mathrm{Au}_{1}^{-}$(peak $\mathrm{A}$ ) and $\mathrm{Au}_{2}^{-}$ (peak B) are emphasized. At the higher photon energy $h \nu=3.14 \mathrm{eV}$, a corresponding analysis yields the spectrum displayed in Fig. 3(b). The relative intensity of feature $\mathrm{B}$ (attributed to emission from $\mathrm{Au}_{2}^{-}$) has increased by a factor of 2.5 .

The branching ratio can also be measured using a standard nanosecond laser. Fig. 3(c) shows the spectrum of $\mathrm{Au}_{3}^{-}$obtained with a photon energy of $h \nu=3.49 \mathrm{eV}$ and a laser pulse length of $6 \mathrm{~ns}$ (third harmonic of a ND-YAG laser). This spectrum, obtained at a considerable higher photon energy (equal to the excitation energy) is similar to that recorded at $3.14 \mathrm{eV}$. This is an indication that at any

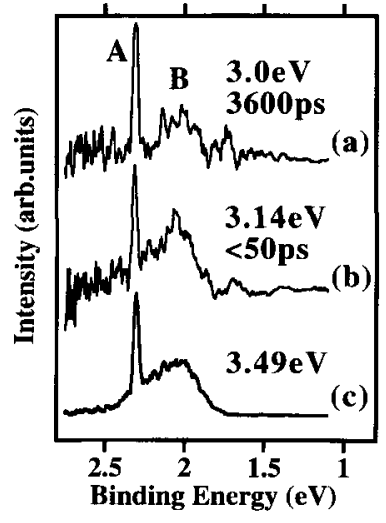

Fig. 5. (a) Difference between the pump/probe spectrum of $\mathrm{Au}_{3}^{-}$ taken at the maximum delay $(\Delta t=3600 \mathrm{ps})$ and at zero delay $(\Delta t=0.0 \mathrm{ps})$. The photon energy of the pump and probe laser pulse is $h \nu=3.0 \mathrm{eV}$. (b) Same difference as in (a), but now the photon energy is changed to $h \nu=3.14 \mathrm{eV}$. (c) Photoelectron spectrum of $\mathrm{Au}_{3}^{-}$recorded with a standard laser (pulse length, $6 \mathrm{~ns}$ ) with a photon energy of $h \nu=3.49 \mathrm{eV}$.

excitation energy significantly higher than $3.14 \mathrm{eV}$, the dissociation occurs with a fixed branching ratio of the two decay channels (eqns (1) and (2)).

Our observations can be explained by the following tentative picture.

- At low photon energies, the decay occurs via a metastable complex with a lifetime that is strongly dependent on the excess energy. At an excitation energy of $3.0 \mathrm{eV}$, the lifetime is $1500 \pm 200$ ps. The complex decays by an unimolecular decay process through a barrier and, accordingly, the lower energy decay channel $\mathrm{Au}_{1}^{-}+\mathrm{Au}_{2}$ has a higher probability. (The electron affinity of $\mathrm{Au}_{1}$ is higher by $0.3 \mathrm{eV}$ than that of $\mathrm{Au}_{2}$. Therefore the decay into $\mathrm{Au}_{1}^{-}+\mathrm{Au}_{2}$ is the lower energy channel [21].)

- At higher photon energy (above $3.14 \mathrm{eV}$ ) the decay occurs directly. The branching ratio does not depend on the photon energy and gives a considerable higher probability for the higher energy channel $\left(\mathrm{Au}_{2}^{-}+\mathrm{Au}_{1}\right)$.

With this example, we have demonstrated the application of femtosecond lasers to photoelectron spectroscopy to study the dynamics of a long-living activated complex, which forms during a chemical reaction. It gives rise to the appearance of a new 
transient feature in the photoelectron spectra. In the future, the use of a higher photon energy of the probe photon (e.g. $h \nu=6 \mathrm{eV}$ ) will yield a full picture of the occupied orbital structure of the transition state analogous to the spectra of the stable species shown in Fig. 1. This new technique of TRPES can not only be used to study various "inverse" chemical reactions but also, for example, catalytic processes on clusters and energy dissipation in nanoparticles.

\section{References}

[1] A.H. Zewail, Femtochemistry, Vols. I, II, World Scientific, Singapore, 1994.

[2] J. Manz, L. Wöste, Femtosecond Chemistry, Vols. I, II, VCH, Weinheim, 1995.

[3] S. Wolf, G. Sommerer, R. Rutz, E. Schreiber, T. Leisner, L. Wöste, R.S. Berry, Phys. Rev. Lett. 74 (1995) 4177.

[4] D.E. Manolopoulos, K. Stark, H.-J. Werner, D.W. Arnold, S.E. Bradforth, D.M. Neumark, Science 262 (1993) 1852.

[5] I.W.M. Smith, Nature 358 (1992) 279.

[6] T.S. Rose, M.J. Rosker, A.H. Zewail, J. Chem. Phys. 88 (1988) 6672

[7] T. Baumert, M. Grosser, R. Thalweiser, G. Gerber, Phys. Rev. Lett. 67 (1991) 3753 .
[8] E.D. Potter, J.L. Herek, S. Pedersen, Q. Liu, A.H. Zewail, Nature 355 (1992) 66.

[9] M. Dantus, M.J. Rosker, A.H. Zewail, J. Chem. Phys. 87 (1987) 2395

[10] X. Song, C.W.Wilkerson Jr., J. Lucia, S. Pauls, J.P. Reilly, Chem. Phys. Lett. 174 (1990) 377.

[11] D.R. Cyr, C.C. Hayden, J. Chem. Phys. 104 (1996) 771.

[12] B. Kim, C.P. Schick, P.M. Weber, J. Chem. Phys. 103 (1995) 6903.

[13] J. Bokor, R. Storz, R.R. Freeman, P.H. Bucksbaum, Phys. Rev. Lett. 57 (1986) 881.

[14] T. Hertel, E. Knoesel, M. Wolf, G. Ertl, Phys. Rev. Lett. 76 (1996) 535.

[15] T. Baumert, R. Thalweiser, G. Gerber, Chem. Phys. Lett. 209 (1993) 29.

[16] I. Fischer, D.M. Villeneuve, M.J.J. Vrakking, A. Stolow, J. Chem. Phys. 102 (1995) 5566.

[17] B.J. Greenblatt, M.T. Zanni, D.M. Neumark, Chem. Phys. Lett. 258 (1996) 523.

[18] B.J. Greenblatt, M.T. Zanni, D.M. Neumark, Science 276 (1997) 1675.

[19] H. Handschuh, Chia-Yen Cha, H. Möller, P.S. Bechthold, G. Ganteför, W. Eberhardt, Chem. Phys. Lett. 227 (1994) 496.

[20] H. Handschuh, G. Ganteför, W. Eberhardt, Rev. Sci. Instrum. 66 (1995) 3838.

[21] J. Hoe, K.M. Ervin, W.C. Lineberger, J. Chem. Phys. 93 (1990) 6987.

[22] H. Handschuh, G. Ganteför, P.S. Bechthold, W. Eberhardt, J. Chem. Phys. 100 (1994) 7093. 